\title{
The Prevalence of Dysphotopsia in Patients with Recent Cataract Surgery
}

\author{
Andreea Dana Fișuș ${ }^{1^{*}}$, Zoltán Madaras ${ }^{1}$, Karin Ursula Horváth ${ }^{1,2}$ \\ 1 Tîrgu Mureș County Hospital, Ophthalmology Clinic \\ 2 University of Medicine and Pharmacy Tîrgu Mureș
}

Pseudophakic dysphotopsia are becoming increasingly important as unwanted side effect after cataract surgery. Objective: The purpose of this study is to compare the photic symptoms experienced by patients after cataract surgery. Material and method: This is a prospective study that included 105 eyes from 99 patients, which underwent uncomplicated phacoemulsification and IOL implantation, between June 2015 and June 2016, performed at Ophthalmology Clinic Tg Mureș. Patients without visually consequential ocular co-morbidity completed a questionnaire, designed to assess subjectively perceived visual functioning and identify symptoms of dysphotopsia. Results: From the total number of patient, hydrophobic lenses were implanted in 95 patients and 10 patients received hydrophilic lenses. Photic effects were reported in $18 \%$ of treated eyes. Although the percentage of dysphotopsia is higher in the hydrophobic lenses category, there was no significant statistical difference between the two categories. Conclusion: The incidence and significance should not be overlooked, thus visual acuity is not enough for evaluating postoperative visual function.

Keywords: dysphotopsia, glare, survey, cataract, intraocular lens

Received 17 August 2016 / Accepted 27 December 2016

\section{Introduction}

The major concerns, nowadays in cataract surgery, are enhancing vision and retina protection against light toxicity. [1-5]

With all the advances in the intraocular lens design, that have improved visual outcome, patient often complain of optical side effects known as dysphotopsia. [4-12]

Negative dysphotopsia represent the absence of vision in the temporal region, located at $20^{\circ}$ from the center, whilst positive dysphotopsia, the presence of glare, halo, or streaks of light. $[5,8,-16]$ Negative dysphotopsia is a more enigmatic symptom.

Glare is caused by the interaction between the stray light and refractive media leading to a reduced contrast sensibility. $[12,17,18]$ Halos (discs of light) are usually perceived in bright light, or foggy weather. $[9,16]$ Thus patient can experience an interference with their mundane activities.

These unpleasant ocular symptoms can appear as early as one week postoperative, and lest up to several months. $[8,11,13,14]$

The mechanism in which this phenomenon is produced is still a matter of much controversy; factors pertaining to lens design are currently debated.

On the one hand these symptoms can often be caused by the optic shapes and diameters of the intraocular lens, current studies shown higher incidence of dysphotopsia correlated with sharp-edge optical design. [5,12,14,1719] Thus negative photopsia is produced by parallel rays of light that pass thru the lenses edge resulting into two

* Correspondence to: Andreea Dana Fișuș

E-mail: fisusandreea@yahoo.com divergent rays that project onto the retina, creating a temporal shadow.[8,10,13,14]

Another possible explanation of dysphotopsia is the interaction between the optical pathway of the eye and the intraocular lens, the mechanism is the reflection of light rays between the optic edge and the anterior capsulorhexis; irrespective of intraocular lens material, nevertheless increased anterior chamber is also know for inducing negative dysphotopsia $[4,6,7,9,14]$

The use of acrylic copolymers as lens material, can also impact the incidence of dysphotopsia.

Hydrophilic acrylic lenses have higher water content then hydrophobic ones, which lead to a higher incident of capsular opacification and a lower incident of dysphotopsia. The higher water content compared of hydrophilic lenses lead to a lower refractive index. A high refractive index is known to cause dysphotopsia.

Aspheric intraocular lenses improve vision by reducing the optical aberration, especially spherical aberration at the retina. These lenses generate a degree of negative spherical aberration thus compensating the positive spherical aberration induced by the cornea. [6,19-22]

Studies show that the blue light-filtering yellow chromophore in some hydrophilic lenses can reduce the effect of positive dysphotopsia, without affecting color vision. [1619] The blue light filtering lenses can improve vision by enhancing contrast, where initially designed to protect the retina form phototoxicity. [16,18,21]

Majority of unpleasant symptoms are transient, thus the "watchful waiting" is the best solution in many cases. Although the majority of symptoms fade in time due to neuroadaptation, if symptoms persist, some measures should 
be taken: first of all eliminating any refractive error with spectacles, then proper diagnosis of dry eye, thus leading to treatment, the use of thick framed glasses may be of use, so is the intermittent use o pupillary dilatation. $[3,5,8,13,14]$

There is a percentage of $1-2 \%$ of patients who present with debilitating, prolonged symptoms, for whom additional surgery may be the solution. Treatment options cited by the current literature are: anterior laser capsulotomy, reverse optic capture, piggy-back intraocular round edge lens in sulcus (reducing the space behind the iris), or iris suture fixation of the capsular bag and lens complex, thus covering or eliminating the anterior capsule. In extreme cases, with debilitating symptoms, lens exchange is discussed. [3-5,8,12-14,18]

The purpose of our study is to determine the incidence of dysphotopsia compared to the type of intraocular lens and examine if there is a correlation between patient satisfaction and the presence of dysphotopsia,

\section{Material and Method}

This is a prospective, observational study that included 105 eyes from 99 patients that underwent uncomplicated phacoemulsification and IOL implantation, between June 2015 and June 2016, performed at Ophthalmology Clinic Tîrgu Mureș.

There is no objective test to diagnose the presence of dysphotopsia

\section{Surgery}

The surgery was performed by two surgeons using the same method, with similar visual outcome. Uneventful standard phacoemulsification was performed; all eyes received an acrylic single-piece intraocular lens in the capsular bag.

\section{Participants}

The patients were divided into categories, based on the IOL type implanted, hydrophilic lenses (Medicontur 640AB and 640ABY) and hydrophobic ones (AcrySof SN60WF and SA60AT). Due to the fact that aspheric lenses have a reduction in optical aberration compared to spherical ones, we only included in the questionnaire phase aspheric acrylic lenses.

The exclusion criteria applied were:

- preoperative condition: retinal or optic nerve damage (macular degeneration, glaucoma neuropathy), corneal or vitreous opacities, amblyopia, corneal astigmatism over 1,25 diopter, dry eye, traumatic cataract, other pathologies that can influence the comprehen- sion of the questionnaire (Alzheimer's disease)

- intraoperative complications(capsular rupture, IOL out of the capsular bag),

- postoperative complications: corneal oedema, significant inflammation, tilted intraocular lens, best corrected vision under 0.1 - unexpected vision loss, cystoid macular oedema, refractive errors $(>1,00$ spherical diopter, or astigmatism). [4,7,19,23]

\section{Questionnaire}

This study was designed to evaluate the photic phenomena from the perspective of subjective multiple question questionnaire, participants were surveyed at the two week postoperative examination. The questionnaire used was a validated questionnaire described in previous studies. [17,23] A single investigator performed all the questionnaires

Our primary outcome measured was regarding patient satisfaction, followed by uncorrected/corrected visual acuity (measured using Sneller chart) and glass independence.

Subjects that complain about dysphotopsia were asked about the nature of these symptoms, and about the way these affect their life. (Table I.)

\section{Results}

The study contained a number of 95 hydrophobic acrylic lenses and 10 hydrophilic lenses.

$86 \%$ of the respondents were satisfied with the postoperative vision.

The mean age of patients in the study is 70.33 , without a significant difference between the two groups, $63 \%$ women and $36 \%$ men.

Dysphotopsias reported at the two week follow-up were present in $24.3 \%$ of cases, $52.63 \%$ of these patients reported the presence of halos and $26.31 \%$ complained of temporal shadow.

Patients were asked to rank the severity of their symptoms, as shown in Fig 1. Sever symptoms were not reported.

The mean for best corrected visual acuity, situated between 0.8-1.0 for both groups was 70.14\% (Fig 2.)

In the study $38.29 \%$ were within the range of $+/$ $0.50 \mathrm{DSph}$ refraction, and $28.08 \%$ within $+/-1,00 \mathrm{DSph}$ refraction.

The presence of dysphotopsia was correlated with the degree of postoperative satisfaction as shown in Fig 3 .

\section{Discussion}

The photic phenomenon is a well known consequence of cataract surgery and pseudophakia. $[3,4,8,10,11,13]$ The

Table I. Questionnaire

\begin{tabular}{|c|c|c|c|c|}
\hline $\begin{array}{l}\text { Postoperative satisfac- } \\
\text { tion grading }\end{array}$ & spectacle independence & $\begin{array}{c}\text { ease of performing mundan activi- } \\
\text { ties (grading) }\end{array}$ & $\begin{array}{c}\text { presence/degree of dyspho- } \\
\text { topsia }\end{array}$ & $\begin{array}{l}\text { willingness conge- } \\
\text { ner ocular surgery }\end{array}$ \\
\hline 1 dissatisfied & near vision yes/no & driving & 0 absent & yes \\
\hline 2/3moderat (neutral) & distance vision yes/no & watching TV/phone/computer & 1 minimal (barely notice) & no \\
\hline 4 satisfied & & reading book/drug prospectus & 2-4 annoying & \\
\hline 5 highly satisfied & & & 5 debilitating & \\
\hline
\end{tabular}




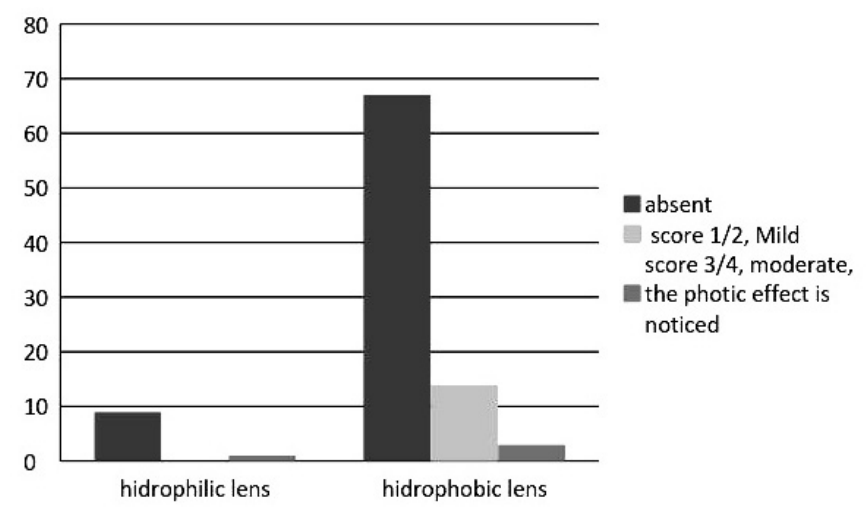

Fig. 1. Grading scale of dysphotopsia

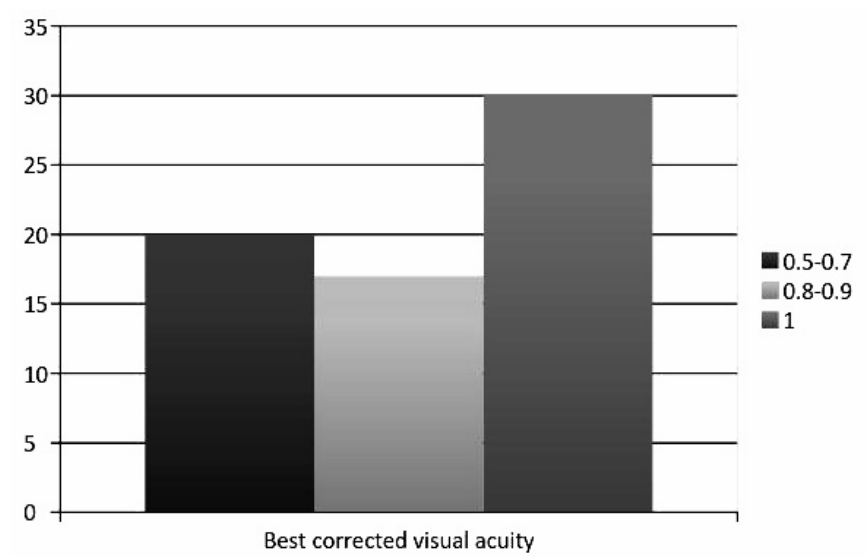

Fig. 2. Postoperative best corrected vision

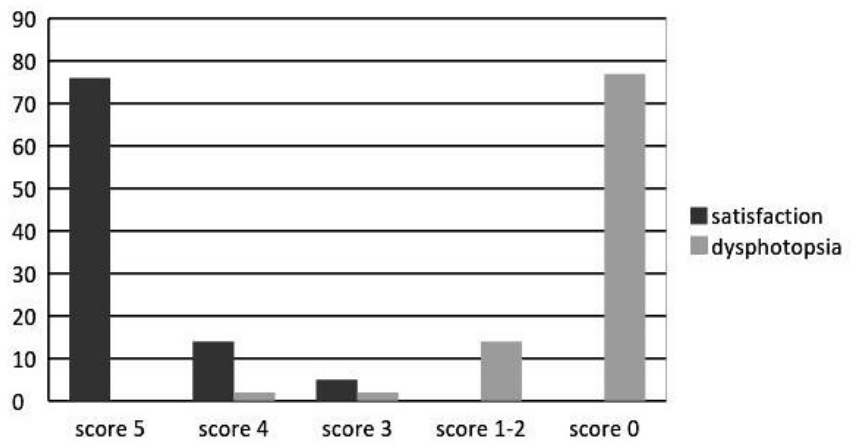

Fig. 3. Patient satisfaction and dysphotopsia presence correlation

simplest way to describe dysphotopsia is that it represent an unwanted patterns on the retina. [5]

The lens visual side effects are reported in the current literature as being between 2 and $18 \%$, with a slight increase for multifocal lenses, were as known, the patients have higher demand. $[8,11,13,14,22]$ The incidence of dysphotopsias in our study is $24.03 \%$, we think that this may be due to our sample size and the phrasing of our question; some questing may have implied an certain answer.

Due to the prevalence of posterior capsule opacification, the study contained more hydrophobic square edge lenses then hydrophilic ones, thus the possible explanation for the high rate of dysphotopsia.

High demand in term of visual outcome, and postoperative satisfaction, make the need of additional chair time. $[8,18,22]$ Thus the postoperative visual acuity is not enough for evaluating the visual function. [5]
Finding these complaints is quite surprising, given that often during the postoperative ocular exam the surgeon finds a good visual acuity. $[9,11]$

Photic phenomena are produced by two major factor lens geometry and ocular physiology. $[5,7,9-14,22]$

Theoretically all the lenses are subjected to some degree of dysphotopsia, as long as they are placed in the capsular bag, nevertheless it is known that hydrophilic lenses are the most commonly used, due to the low incidence of dysphotopsia, although the lens material makes it prone for the development posterior capsular opacification. [4,7,20-22]

When conducting a subjective questionnaire, minor changes in the way questions are phrased can change the outcome. We observed when asking broad questions the patients tend not to mention dysphotopsia symptoms, when the questions were explained the number of patients with visual complaints drastically changed. As a result we preferred the direct inquiry. This can also explain the high number of patients with dysphotopsia compared with the high degree of satisfaction.

While we did not find a correlation between the type of intraocular lens implanted, nor the presence of sharp-edge lens, and the presence and severity of dysphopsia, it is currently known that there is a link, thus additional studies should be conducted.

Due to the careful selection of the patients, one of the weak points of our study is the lack of reaching the statistical significant, we think that this can be solved by enlarging the sample size, and including lenses that are not exact replicas of one another.

What we found interesting is that we did not determine a correlation between the objectified outcome of the patient (presence of dysphotopsia) and patient dissatisfaction. One answer can be that the vision before the surgery was very low, thus making any increase in vision a definite increase in patient satisfaction.

Although many patients experienced some degree of dysphotopsia, only a few reported them as being bothersome, or even mentioned them before the deliberate questioning, thus leading to the conclusion that additional time for postoperative consultation is needed to fully understand the extent of these symptoms. Case by case treatment is required. [9]

After extensive literature review what we found is that with all the studies conducted in this department, there isn't a way to predict who will experience dysphotopsia. [10]

In order to minimize the prevalence of dysphotopsia surgeons should opt for unfinished or frosted lens edge, or materials with low index of reflection. $[6,9,12]$

In the current state, the surgeon has a wide range of IOL to chose from, as regarding the materials used, refractive and additional profiles, nevertheless, we would like to point out that due to the enigmatic mechanism, patient related factors play an equally important role (idiosyncratic predisposition, prominent globe, shallow orbit) $[5,22]$ 


\section{Conclusion}

The optical side effects gradually gained ground, being the major postoperative concern.

When choosing the right intraocular lens for the patient it is important to inquire about his/hers daily activities, which can be an indicator about the tolerance of possible dysphotopsia.

Treatment should be adequately timed because the symptoms usually fade in time due to capsular fibrosis, cortical adaptation, or the patient final compromise.

Photic phenomena are produced by two major factor lens geometry and ocular physiology.

Placing the lens haptics horizontally appears to reduce the presence of negative dysphotopsia.

Although hydrophilic lenses have a lower incidence of dysphotopsia than hydrophobic ones, when choosing the right intraocular lens it should be known that hydrophilic lenses have a higher incidence of posterior capsule opacification.

\section{Conflict of interest}

None to declare.

\section{References}

1. Vlăduțiu C, Chiseliță D, Voinea L, Tătaru C, Filip M, Baltă F, et al. Cristalinul, Popescu I, Ciuce C: Tratat de chirurgie. Academia Română, București, 2013;111-195

2. Yanoff M, Duker JS. Cataract: Ophthalmology. Mosby, Spain, 2004, 37:280-282/ Lens replacement 40:293-308/ Patient work-up for cataract surgery 43:327-330

3. Masket S, Fram N. Pseudophakic negative dysphotopsia: surgical management and new theory of etiology. J Cataract refract Surg. 2011; 37:1199-1207

4. Jafarinasab MR, Feizi S, Baghi AR, Ziaie M, Yaseri M. Aspheric versus Spherical Posterior Chamber, Intraocular Lenses. J Ophthalmic Vis Res. 2010; 5 (4): 217-222

5. Davison JA, Cunha JP, Patel AS, Schwiegerling J, Muftuoglu O. Recent studies provide an updated clinical perspective on blue light-filtering IOLs. Graefes Arch Clin Exp Ophthalmol. 2011; 249:957-968

6. Holladay JT, Zhao H, Reisin C. Negative dysphotopsia: The enigmatic penumbra. J Cataract Refract Surg. 2012; 38(7): 1251-1265

7. Tang $\mathrm{Y}$, Song $\mathrm{H}$, Chen J, Tang $\mathrm{X}$. Comparison of pseudophakic retinal straylight in spherical/aspherical and hydrophobic/hydrophilic intraocular lens. Int J Ophthalmol. 2015; 8(6):1146-1150

8. Makhotkina NY, Berendschot TJM, Beckers HJM, Rudy M, Nuijts A Treatment of negative dysphotopsia with supplementary implantation of a sulcus-fixated intraocular lens. Graefes Arch Clin Exp Ophthalmol. 2015; 253:973-977

9. Radmall BR, Floyd A, Oakey Z, Olson RJ. Refractive index and its impact on pseudophakic dysphotopsia. Clinical Ophthalmology. 2015; 9:1353-1358

10. Wenzel M, Menapace R, Eppig T, Langenbucher A. Is the Memory Effect of the Blind Spot Involved in Negative Dysphotopsia after Cataract Surgery. Journal of Ophthalmology. 2015; doi.org/10.1155/2015/786579

11. Katsev DA. Dysphotopsia and Multifocal IOLs. CRST. 2012; 5:28-30

12. Pace NL, Samore M, Olson RJ. Dysphotopsia in phakic and pseudophakic patients: Incidence and relation to commonly used intraocular lenses. J Cataract Refract Surg. 2015; 26(6): 810-6

13. Vamosi P, Csakany B, Nemeth J. Intraocular lens exchange in patients with negative dysphotopsia symptons. J Cataract Refract Surg. 2010; 36(3):418-424, DOI: 10.1016/j.jcrs.2009.10.035

14. Osher $\mathrm{RH}$, Negative dysphotopsia: long-term study and possible explanation for transient symptoms. J Cataract Refract Surg. 2008; 34(10):1699-1707

15. Caceres V. Treating, eliminating negative dysphotopsia. EW. 2014; 19(4):38-40

16. Beiko $\mathrm{GHH}$. A pilot study to determine if intraocular lens choice at the time of cataract surgery has an impact on patient-reported driving habits. Clinical Ophthalmology. 2015;9:1573-1579

17. Tester R, Olson RJ, Pace LN, Samore M. Dysphotopsia in phakic and pseudophakic patients: Incidence and relation to commoly used intraocular lenses. J Cataract Refract Surg. 2000; 26(6):810-816

18. Hammond BR Jr, Renzi LM, Sachak S, Brint SF. Contralateral comparison of blue-filtering and non-blue-filtering intraocular lenses: glare disability, heterochromatic contrast, and photostress recovery. Clinical Ophthalmology. 2010; 4:1465-1473

19. Guo Y, Li J, Song H, Tang X. Comparison of the Retinal Straylight in Pseudophakic Eyes with PMMA, Hydrophobic Acrylic and Hydrophilic Acrylic Spherical Intraocular Lens. Journal of Ophthalmology. 2014; dx.doi.org/10.1155/2014/340759

20. Li Y, Wang J, Chen Z, Tang X. Effect of Hydrophobic Acrylic versus Hydrophilic Acrylic Intraocular Lens on Posterior Capsule Opacification: Meta-Analysis. 2013; 8(11)doi:10.1371/journal.pone.0077864

21. Xi L, Liu Y, Zhao F, Chen $\mathrm{CH}$, Cheng B. Analysis of glistenings in hydrophobic acrylic intraocular lenses on visual performance. Int $J$ Ophthalmol. 2014; 7(3):446-451

22. van der Linden JW, van der Meulen I, Mourits MP, Lapid-gortzak R. Comparison of a hydrophilic and a hydrophobic apodized diffractive multifocal intraocular lens. Int Ophthalmol. 2013; 33:493-500

23. Vyas A. Incidence of Dysphotopsia in Patients Implanted with the C-flex® Intraocular Lens with $360^{\circ}$ Enhanced Edge: A QuestionnaireBased Study. J Clin Exp Ophthalmol. 2015; 6:1. doi.org/10.4172/21559570.1000391 